\title{
Deep vein thrombosis after spine operation in prone position with subclavian venous catheterization
} - a case report-

\author{
Jae Kyung Cho, Jin Hee Han, Sung Wook Park, and Keon Sik Kim \\ Department of Anesthesiology and Pain Medicine, Kyung Hee University College of Medicine, Seoul, Korea
}

We experienced a case of deep vein thrombosis after spine surgery in the prone position with a central venous catheter (CVC). Posterior lumbar interbody fusion was performed on a 73-year-old female patient who was diagnosed with spinal stenosis. Accordingly, in the operation room under general anesthesia, two-lumen CVC were inserted into the left subclavian vein. The surgery was performed in the prone position with a Wilson frame. On the next day, there was a sudden occurrence of severe edema in the patient's left arm. By ultrasonography and computed tomography scanning, extensive deep vein thrombosis was observed in the left subclavian vein. The existence of a factor affecting blood flow such as the prone position may increase the risk of thrombus formation. Therefore, careful perioperative evaluation should be implemented. (Korean J Anesthesiol 2014; 67: 61-65)

Key Words: Central venous catheters, Prone position, Upper extremity deep vein thrombosis.

In anesthesia for lumbar spine surgery, usually a change in position of the patient from the supine position to the prone position is required. Patients in the prone position under general anesthesia generally develop hemodynamic changes [1]. In several studies, changes in cardiac function after prone positioning were measured, with venous return and ventricular compliance showing a reduction [2].

Central venous catheterization (CVC) in the operation room is primarily carried out under general anesthesia, for the purpose of central venous pressure monitoring as well as for gaining access to injection channels for drugs and IV lines. Potential complications that can be caused by CVC are artery puncture, hematoma, pneumothorax, hemothorax, infection, and vein thrombosis [3]. In this paper, the authors would like to report a case of postoperative venous thrombosis that occurred after spinal surgery on a patient in the prone position, with a CVC via the subclavian vein.

\section{Case Report}

A 73-year-old female patient was diagnosed with spinal stenosis on L1-L5, and it was decided to perform posterior lumbar

Received: April 23, 2013. Revised: 1st, May 27, 2013; 2nd, July 5, 2013; 3rd, July 10, 2013; 4th, July 11, 2013. Accepted: July 11, 2013.

Corresponding author: Sung Wook Park, M.D., Department of Anesthesiology and Pain Medicine, Kyung Hee University College of Medicine, 26, Kyungheedae-ro, Dongdaemun-gu, Seoul 130-872, Korea. Tel: 82-2-958-8589, Fax: 82-2-958-8580, E-mail: demerol@khmc.or.kr (c) This is an open-access article distributed under the terms of the Creative Commons Attribution Non-Commercial License (http:// creativecommons.org/licenses/by-nc/3.0/), which permits unrestricted non-commercial use, distribution, and reproduction in any medium, provided the original work is properly cited. 
spinal fusion. The patient had height of $154 \mathrm{~cm}$ and weight of $72 \mathrm{~kg}$. Except for hypertension, no other specific medical history was known. Prior to surgery, there were no abnormal findings in the complete blood count, and electrolyte and blood coagulation tests.

Before anesthetic induction, the patient's blood pressure was $150 / 70 \mathrm{mmHg}$, heart rate was 82 beats/min, and oxygen

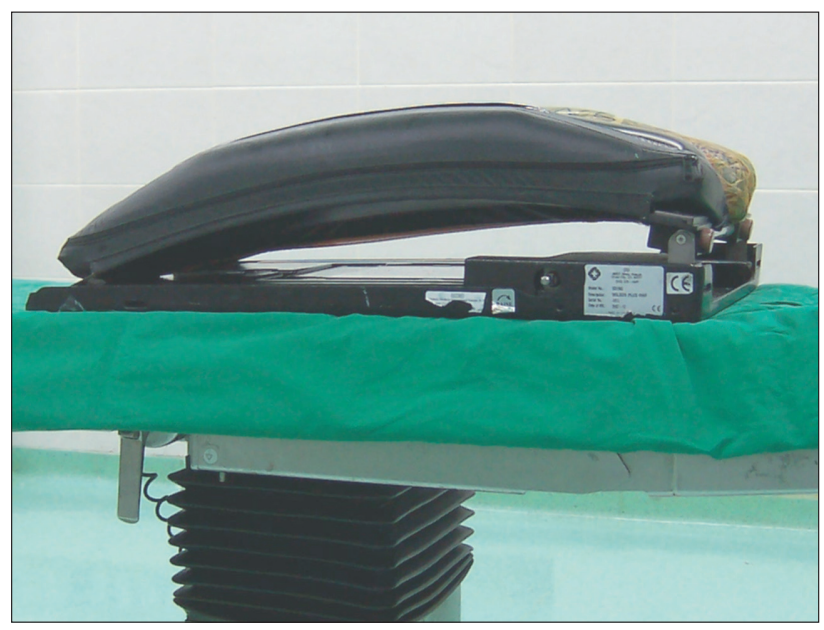

Fig. 1. Wilson frame (OSI, Union City, CA, USA). saturation was $98 \%$. Anesthetic induction was conducted with injection of propofol $120 \mathrm{mg}$ and rocuronium $50 \mathrm{mg}$, followed by endotracheal intubation. General anesthesia was maintained through sevoflurane (1-1.5 \%)- $\mathrm{O}_{2}(2 \mathrm{~L} / \mathrm{min})-\mathrm{N}_{2} \mathrm{O}(2 \mathrm{~L} / \mathrm{min})$. For direct measurement of the arterial pressure, right radial artery cannulation was performed. It was planned that the patient would be managed in the intensive care unit for a few days after the surgery, and at the request of the orthopedic surgeon, a subclavian venous catheter, which is comparatively convenient for long term maintenance, was to be inserted. Due to a skin lesion in the right subclavian area, central venous catheter insertion was conducted in the left subclavian vein with a two-lumen CVC set (Arrow International, Inc., Reading, PA, USA). The catheter was fixed at a depth of $15 \mathrm{~cm}$ while the intravenous supply after blood regurgitation was confirmed. Afterwards, the patient was turned into the prone position on the Wilson frame (OSI, Union City, CA, USA) for the surgery (Fig. 1). After changing the position, the upper chest area from above the nipples and including the subclavian area showed overall compression. In order to prevent pressure by compression, a surgical cotton pad was applied to the applicable area, and free flow of fluid through the CVC was checked before commencing the operation. During the whole operating time of 4 hours and 45 minutes, the mean arterial blood pressure and the heart rate were maintained at 65-75
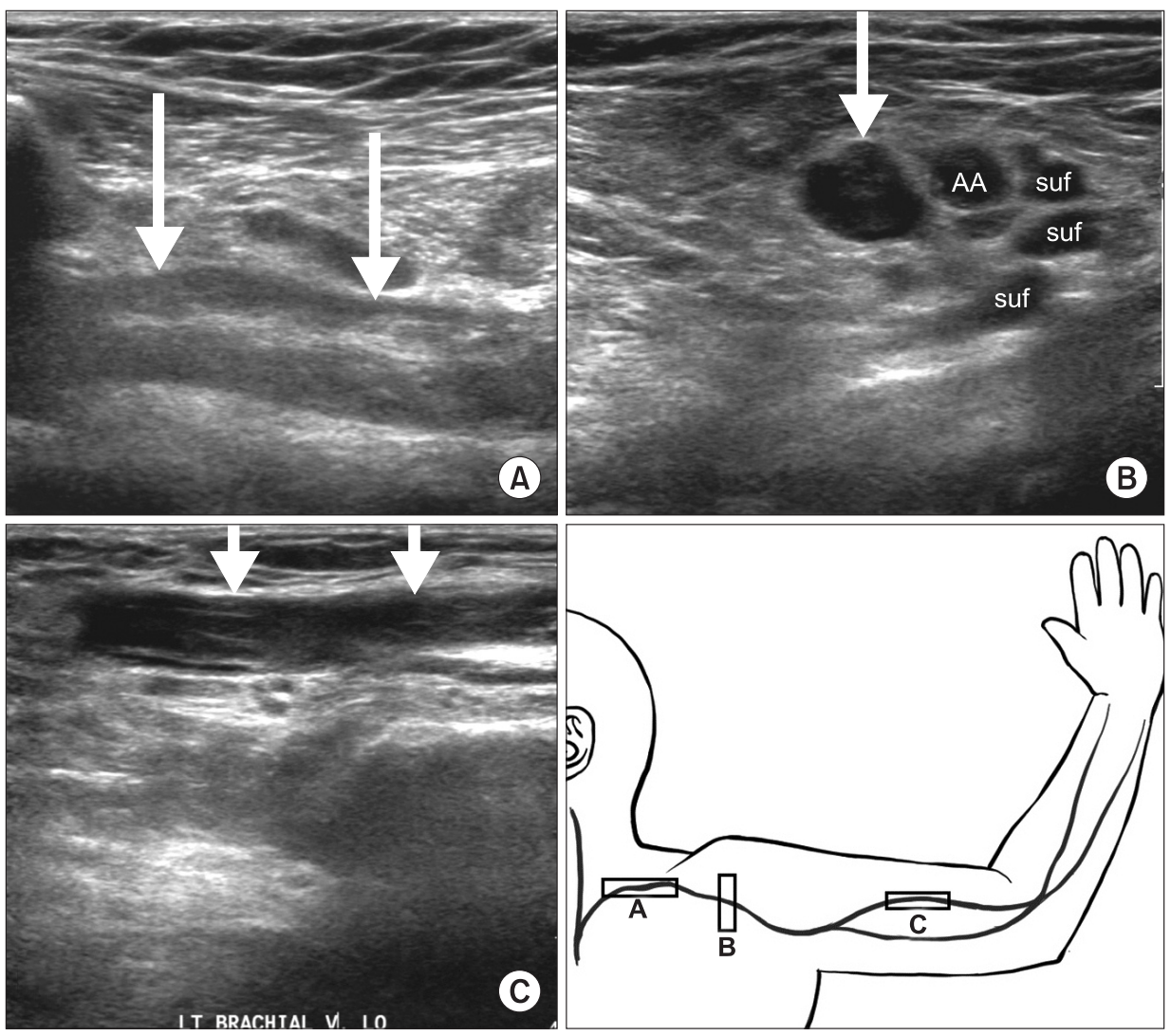

Fig. 2. Left upper extremity ultrasonographic images and anatomy diagram. Extensive thrombosis (arrows) is shown at (A) subclavian, (B) axillary and (C) brachial veins (AA: axillary artery, Suf: expanded superficial veins of the axilla). 
$\mathrm{mmHg}$ and 60-75 beats/min, respectively, showing stable vital signs. Total fluid input during the surgery amounted to $1,200 \mathrm{ml}$ of normal saline, $500 \mathrm{ml}$ of colloid, 5 units of packed red blood cell and 3 units of fresh frozen plasma, and the actual blood loss amounted to $1,500 \mathrm{ml}$ and the urine output was $850 \mathrm{ml}$. After the operation, the patient was transferred to the intensive care unit. The patient was mentally alert, with a blood pressure of $130 / 80 \mathrm{mmHg}$ and heart rate of 61 beats/min. In the blood coagulation test carried out immediately after the operation, the prothrombin time (INR) was measured at 1.13 and activated partial thromboplastin time was 42.3 seconds.

On the next day, there was a sudden occurrence of severe edema in the patient's left arm, and the fingertips of her left hand felt cold. The CVC was removed from the patient immediately.
A blood test conducted on the day of the symptom showed that D-dimer had increased above $20 \mathrm{~g} / \mathrm{ml}$, which led to the suspicion of deep vein thrombosis. To suppress the swelling, the patient's left arm was wrapped with a pressure bandage, and ultrasonography and computed tomography scan were performed on the patient. On ultrasonography, extensive deep vein thrombosis was observed in the left subclavian vein and caudal branch (Fig. 2) while on computed tomography, thrombosis was found in the left brachiocephalic vein and subclavian vein (Fig. 3). Immediately, thrombolysis was conducted in the upper left arm through a venogram (Fig. 4). Over the next 3 days after the thrombolysis, anticoagulation therapy was carried out by concurrently administering heparin $500 \mathrm{IU} / \mathrm{h}$ and urokinase 100,000 IU/h, and over the next 6 days, urokinase was stopped and only heparin was
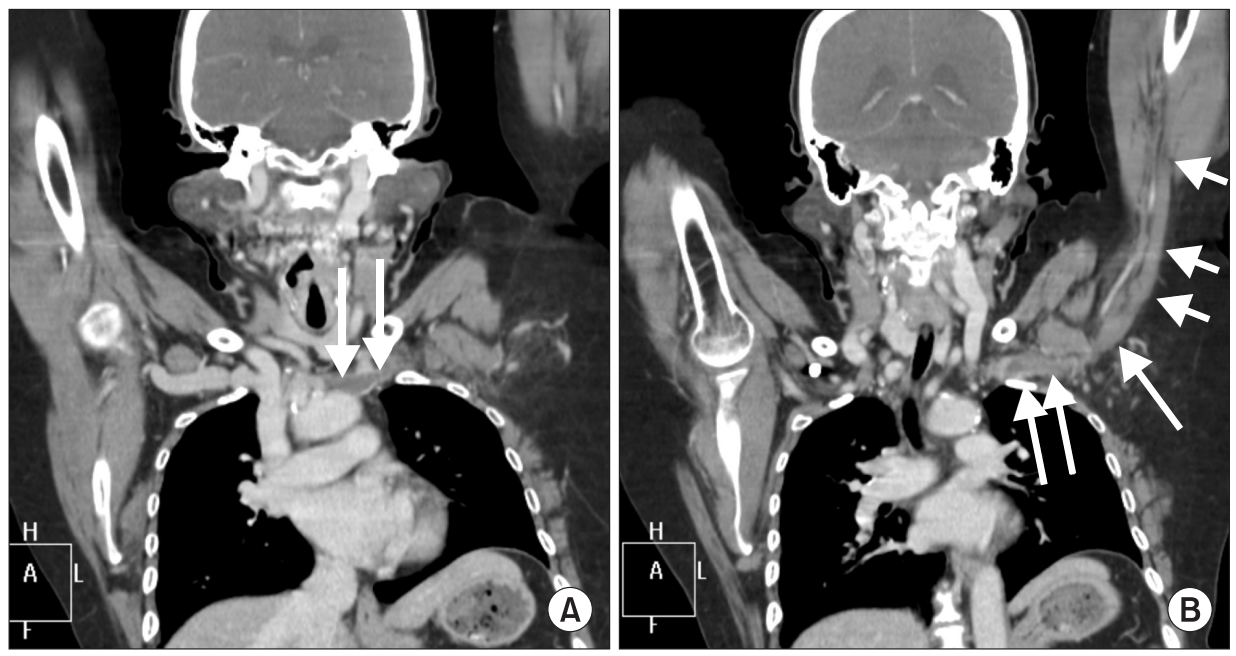

Fig. 3. Coronal multiplanar reformatting images show diffuse extensive thrombosis (arrows) at (A) left brachiocephalic, (B) subclavian, axillary and brachial veins.
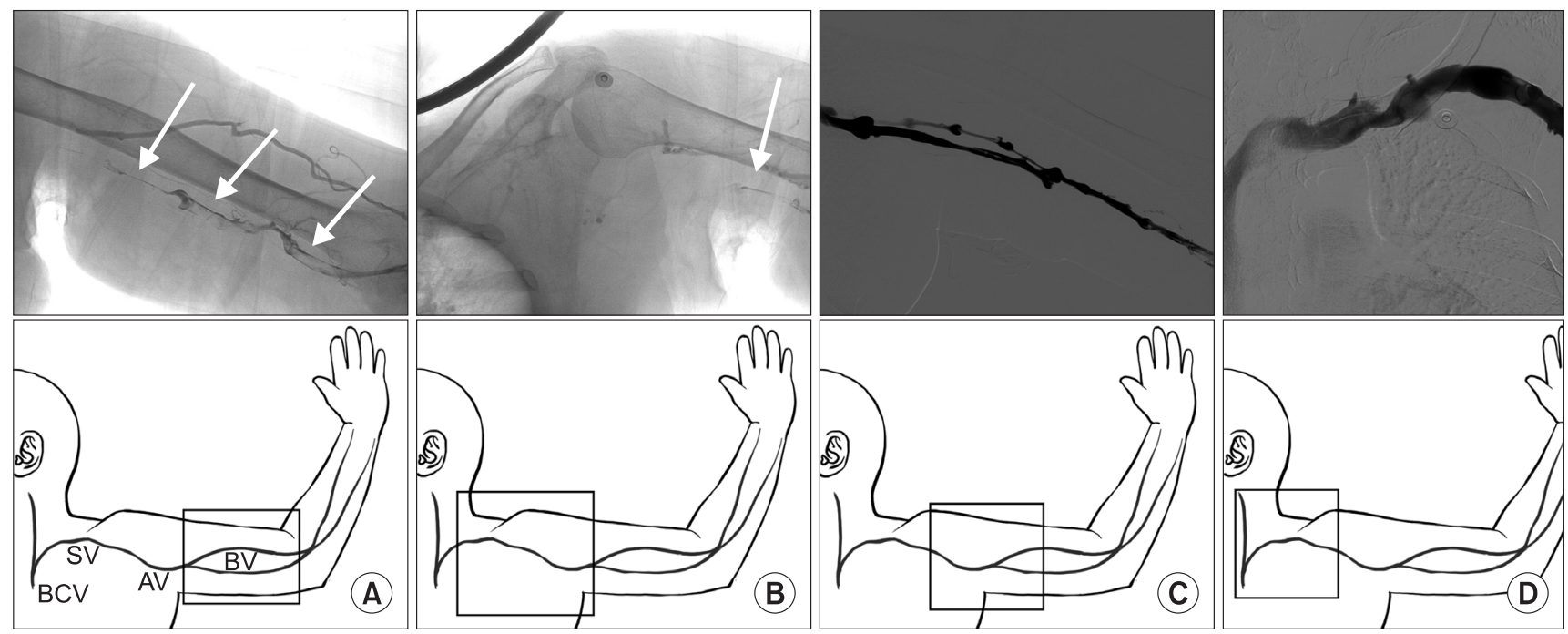

Fig. 4. Pre-thrombolysis venogram images (A, B) show diffuse, extensive thrombosis (arrows) at left brachial, axillary, subclavian and brachiocephiliac veins. Post-thrombolysis venogram images $(C, D)$ show that previous extensive thrombosis in the left upper extremity veins has disappeared completely (BCV: brachiocephalic vein, SV: subclavian vein, AV: axillary vein, BV: brachial vein). 
administered. The patient showed no more particular complications, and was discharged from the hospital 2 weeks after the treatment.

\section{Discussion}

Deep vein thrombosis refers to venous blood congestion creating thrombosis in the deep vein. Of the clinically manifest types of deep vein thrombosis, those related to the central vein have been reported to be approximately 0 to $12 \%$ [4]. Surgeryrelated risk factors for deep vein thrombosis include duration of surgery, the prone position, lumbar vertebral surgery, blood loss and bed rest period [5]. Although deep vein thrombosis can give rise to pulmonary thromboembolism which can lead to death, it is a disease that is difficult to diagnose because its symptoms are usually atypical $[6,7]$. Therefore surgery requires advance identification of the risk factors that can cause deep vein thrombosis. In this case, the patient's risk factors for deep vein thrombosis included smoking, long surgery time (over three hours), prone position during surgery, lumbar vertebral surgery, and massive blood loss (above 1,500 ml).

In particular, the surgery performed in the prone position over long hours seems to have acted as a significant risk factor of deep vein thrombosis. This is due to the fact that the speed of arterial blood circulation may decrease in the prone position, and the reduced speed of deep vein blood circulation is a core factor in the formation of deep vein thrombosis [1,8]. Among the tables used for spine surgery, a higher tendency for reduced cardiac performance was measured with the Wilson frame due to reduced venous return $[1,2]$. In this case, it may be inferred that the reduced venous return may have affected formation of the thrombus. Therefore, in the case of the prone position, use of an open-frame table such as a Jackson spinal table which can relatively minimize an increase in the intrathoracic pressure and intraabdominal pressure should be considered, and in the case of unavoidable use of a closed-frame table such as the Wilson frame, the use of a cushion or padding should be considered in order to minimize the pressure applied to each part of the body.

Joffe et al. [9] reported that the CVC is the most significant predictive indicator for deep vein thrombosis in the upper limbs. In this case, it appears that the existence of a contaminant such as a CVC accelerates thrombosis formation when the patient has already been exposed to reduced speed of blood circulation in the vein due to pressure from the prone position during an operation [10]. Routes which can be selected as insertion points of CVC can be largely divided into the jugular vein, subclavian vein and femoral vein. In the case of the femoral vein, there is a higher risk of infection and occurrence of mechanical complications compared to other routes, and therefore selection is generally made out of the jugular vein or subclavian vein for insertion of a CVC [3]. The subclavian vein is more convenient than the internal jugular vein for long term maintenance of CVC, and some research results report that there is a higher risk of CVCrelated infection with the internal jugular vein than the subclavian vein [11]. The authors decided on insertion of a subclavian catheter by consulting with the surgeon after consideration of such factors. However, as in this case, considering the pressure which can be applied to the subclavicular area in the prone position and the consequent thrombotic risk, more caution must be taken in selecting the route for CVC insertion.

There are still some controversies over the proper treatment for upper limb deep vein thrombosis related to central venous catheters due to the lack of randomized controlled trials [12,13]. Currently, the most common treatments include anticoagulation therapy with low molecular weight heparin and a vitamin $\mathrm{K}$ antagonist while other methods include thrombolysis, surgery, thrombectomy and balloon dilatation. Each of these methods has its pros and cons. Anticoagulation therapy entails the risk of bleeding and the other methods entail possible induced complications as they are invasive treatment, requiring caution thereto.

It is also clinically very important to determine the time of removal of the catheter from the patient with an upper limb deep vein thrombosis. According to the guidelines of the American College of Chest Physicians, it is recommended that the catheter be removed 3-5 days after anticoagulation therapy for CVC-related thrombosis patients who no longer need CVC [14]. However, some researchers argue that immediate removal of the central venous catheter can be more beneficial to the patient [15]. In this case, the removal of the CVC took place immediately after symptoms of thrombosis occurred, but considering the possible development of complications, a more prudent approach might be required for timing the catheter removal.

The authors report one case of deep vein thrombosis related to CVC in a patient who received surgery in the prone position. In this situation, the speed of the deep vein blood circulation was reduced, and the slowed blood circulation may have expedited thrombus formation. In order to prevent thrombus formation, more careful attention must be given to the patient's position during surgery, especially if factors exist that could affect the blood flow in blood vessels such as CVC. 


\section{References}

1. Yokoyama M, Ueda W, Hirakawa M, Yamamoto H. Hemodynamic effect of the prone position during anesthesia. Acta Anaesthesiol Scand 1991; 35: 741-4.

2. Dharmavaram S, Jellish WS, Nockels RP, Shea J, Mehmood R, Ghanayem A, et al. Effect of prone positioning systems on hemodynamic and cardiac function during lumbar spine surgery: an echocardiographic study. Spine (Phila Pa 1976) 2006; 31: 1388-93.

3. McGee DC, Gould MK. Preventing complications of central venous catheterization. N Engl J Med 2003; 348: 1123-33.

4. Timsit JF, Farkas JC, Boyer JM, Martin JB, Misset B, Renaud B, et al. Central vein catheter-related thrombosis in intensive care patients: incidence, risks factors, and relationship with catheter-related sepsis. Chest 1998; 114: 207-13.

5. Ferree BA, Stern PJ, Jolson RS, Roberts JM 5th, Kahn A 3rd. Deep venous thrombosis after spinal surgery. Spine (Phila Pa 1976) 1993; 18: 315-9.

6. Wilson WC, Frankville DD, Maxwell W, Carpenter T, Hastings RH. Massive intraoperative pulmonary embolus diagnosed by transesophageal echocardiography. Anesthesiology 1994; 81: 504-8.

7. Scarvelis D, Wells PS. Diagnosis and treatment of deep-vein thrombosis. CMAJ 2006; 175: 1087-92.

8. Nagahiro I, Watanuki Y, Sato S, Nakashima A. Venous velocity of the right femoral vein decreases in the right lateral decubitus position compared to the supine position: a cause of postoperative pulmonary embolism? Acta Med Okayama 2007; 61: 57-61.

9. Joffe HV, Kucher N, Tapson VF, Goldhaber SZ. Upper-extremity deep vein thrombosis: a prospective registry of 592 patients. Circulation 2004; 110: 1605-11.

10. Mai C, Hunt D. Upper-extremity deep venous thrombosis: a review. Am J Med 2011; 124: 402-7.

11. Lorente L, Henry C, Martín MM, Jiménez A, Mora ML. Central venous catheter-related infection in a prospective and observational study of 2,595 catheters. Crit Care 2005; 9: R631-5.

12. Flinterman LE, Van Der Meer FJ, Rosendaal FR, Doggen CJ. Current perspective of venous thrombosis in the upper extremity. J Thromb Haemost 2008; 6: 1262-6.

13. Baskin JL, Pui CH, Reiss U, Wilimas JA, Metzger ML, Ribeiro RC, et al. Management of occlusion and thrombosis associated with longterm indwelling central venous catheters. Lancet 2009; 374: 159-69.

14. Albers GW, Amarenco P, Easton JD, Sacco RL, Teal P; American College of Chest Physicians. Antithrombotic and thrombolytic therapy for ischemic stroke: American College of Chest Physicians Evidence-Based Clinical Practice Guidelines (8th Edition). Chest 2008; 133(6 Suppl): S630-69.

15. Margey R, Schainfeld RM. Upper extremity deep vein thrombosis: the oft-forgotten cousin of venous thromboembolic disease. Curr Treat Options Cardiovasc Med 2011; 13: 146-58. 